\title{
O USO DE RADIAÇÃo ULTRAVIOLETA PARA O PRÉ-TRATAMENTO DE AMOSTRAS EM ANÁLISE INORGÂNICA
}

\author{
Andrea Cavicchioli e Ivano Gebhardt Rolf Gutz* \\ Instituto de Química, Universidade de São Paulo, Av. Prof. Lineu Prestes, 748, 05508-900 São Paulo - SP
}

Recebido em 28/11/02; aceito em 15/4/03

\begin{abstract}
USE OF UV RADIATION FOR SAMPLE PRETREATMENT IN INORGANIC ANALYSIS. The basic principles of UV irradiation as sample pre-treatment step and its potential for inorganic analysis are illustrated and discussed through significant examples from the literature. Not only does this overview cover the classical applications of this technique in the decomposition of organic matter in electroanalysis, but it also presents recent trends, including the increasing interest in employing UV irradiation in flow analytical systems, successful attempts to enhance its effectiveness and the coupling with chromatographic and spectroscopic methodologies. Furthermore, a number of relevant cases of UV-driven derivatization reactions involving photo-sensitive inorganic species are presented, showing some convenient options to perform fast and reliable determination of inorganic and organic analytes.
\end{abstract}

Keywords: UV radiation; sample pretreatment; inorganic analysis.

\section{INTRODUÇÃO}

Com a expressão pré-tratamento de amostra se indica o conjunto dos procedimentos necessários para converter física e quimicamente uma amostra em uma forma que permita efetuar, dentre as limitações impostas pela natureza e a morfologia da mesma, a determinação do(s) analito(s) e realizar sua quantificação o quanto mais precisa e exata ${ }^{1-4}$.

Considerando o vasto panorama da química analítica, em particular a multiplicidade de tipos de amostras e toda a ampla gama de técnicas de detecção, sem contar os inúmeros possíveis analitos, a etapa de pré-tratamento da amostra pode incluir os mais diversos processos, freqüientemente vários deles em seqüências que, amiúde, dão origem a procedimentos integrais bastante demorados e complexos. A título de exemplo, veja-se o recente trabalho de Hanway et $a l .{ }^{5}$ sobre um procedimento para a quantificação de adutos benzenocisteína presentes nas cadeias protéicas da hemoglobina (a globina), apontados como marcadores biológicos da exposição da população a hidrocarbonetos aromáticos. O tempo para realizar a detecção por GC-MS constitui cerca de $0,2 \%$ da duração total do ensaio, que inclui as etapas de diálise, precipitação e purificação da proteína, hidrólise, purificação por extração em fase sólida, duas separações por HPLC e finalmente a derivatização para o derivado sililado, prévia à injeção no cromatógrafo para gases. Trata-se de exemplo extremo, mas bastante emblemático, da variedade de possíveis processos envolvidos e da relevância do preparo da amostra para alcançar objetivos analíticos às vezes árduos.

De fato, porém, para simplificar a questão, não é impróprio afirmar que as operações de preparo da amostra se restringem, na quase totalidade, a poucas classes de operações: solubilização, transferência de meio, pré-concentração/diluição, purificação, derivatização. A solubilização de matrizes sólidas é freqüente em amostras como alimentos, sedimentos, tecidos biológicos, solos, rochas, etc.. A transferência de analitos de um meio para outro inclui sua extração da fase sólida (p. ex., metais em sedimentos) ou gasosa (p. ex., determinação de $\mathrm{SO}_{2}$ ) para a fase aquosa onde normalmente é efetuada a

*e-mail: gutz@iq.usp.br determinação. Já a transferência de um solvente para outro é bastante comum nas separações por extração líquida, como em análises cromatográficas e eletroforéticas. A partição líquido-sólido em colunas ou a deposição eletroquímica exemplificam a pré-concentração de analitos, fundamental na determinação de traços abaixo do limite de determinação das técnicas. Por derivatização entende-se a conversão quantitativa do analito em uma forma química mais apropriada para a detecção, servindo de ilustração: a formação de complexos coloridos de íons inorgânicos para espectrofotometria; a conversão de metais em hidretos em algumas versões da absorção atômica; ou ainda a transformação em espécies eletroativas de analitos não sujeitos a detecção por sensores eletroquímicos.

A exigência da eliminação de interferentes impõe-se com muita frequiência, dependendo de muitos fatores mas, particularmente, do tipo de técnica de detecção. A irradiação da amostra por ondas eletromagnéticas da região da ultravioleta pode ser útil tanto na decomposição de interferentes, tais como substâncias orgânicas, como na derivatização de analitos. As técnicas eletroanalíticas são particularmente vulneráveis à presença de material orgânico dissolvido (“dissolved organic matter, DOM"). Isto acontece quer porque este gera sinais espúrios ou afeta a linha base, reduzindo assim a relação sinal/ruído, quer porque interage com o analito, levando à formação de adutos, eventualmente, eletroquimicamente inertes ${ }^{6}$. No entanto, os devidos cuidados devem ser tomados também com aquelas técnicas que, por serem inerentemente mais destrutivas (isto é, as de espectroscopia atômica em elevadas temperaturas como AA e ICP, em todas as suas variantes), sofrem menor influência da matriz orgânica, ao menos em princípio.

O termo derivatização foi escolhido para incorporar numa única categoria, processos de conversão química do analito em uma forma apropriada para possibilitar ou otimizar uma determinada técnica de detecção. As vantagens do uso de reações fotoquímicas foram resumidas com clareza por Pérez-Ruiz et al. ${ }^{7}$ : não requerem necessariamente a adição de reagentes nem, portanto, bombas, pontos de confluência e células de mistura, em operação em fluxo; as reações são geralmente de tipo radicalar e ocorrem em tempos mais curtos do que as reações térmicas convencionais; podem se tornar extremamente seletivas, restringindo-se o espectro incidente através de filtros e monocromadores. 
Nas próximas seções, após um parágrafo introdutório dirigido a um público de químicos analíticos, sobre alguns aspectos teóricos e práticos básicos dos processos fotoquímicos, procurar-se-á mostrar as potencialidades do uso da radiação ultravioleta no tratamento prévio de amostras através de exemplos representativos, sem a pretensão de uma revisão exaustiva da matéria. Processos fotoquímicos de decomposição e de derivatização serão abordados separadamente, distinguindo entre procedimentos bem conhecidos e estabelecidos na prática analítica, e as novas tendências desta área.

\section{ALGUNS ASPECTOS TEÓRICOS E PRÁTICOS SOBRE PROCESSOS FOTOQUÍMICOS}

A radiação ultravioleta cobre a região do espectro eletromagnético entre 400 e $100 \mathrm{~nm}$. O Comitê Internacional da Iluminação ("Commission Internacional de 1'Eclairage") recomenda a classificação em UV-A (400-315 nm), UV-B (315-280) e UV-C (280-100 $\mathrm{nm})^{8}$, sendo que, amiúde, se refere à primeira faixa como ultravioleta próximo e à última, como ultravioleta extremo ou de vácuo. A denominação de ultravioleta de vácuo deve-se à necessidade de, ao se operar em baixos comprimentos de onda, remover o $\mathrm{O}_{2}$ atmosférico que absorve radiação em $\lambda<200 \mathrm{~nm}^{9}$.

Na prática, o aproveitamento do espectro ultravioleta é limitado pelos três tipos de fontes disponíveis comercialmente: lâmpadas de deutério, xenônio e vapor de mercúrio (Figuras 1 e 2). Entre elas, as de vapor de mercúrio são as mais usadas em aplicações de laboratório e industriais, quando não é exigida muita estabilidade (no que as lâmpadas de deutério são mais favoráveis) ou amplo leque de emissão, cobrindo também o visível e infravermelho próximo (propiciado pelas lâmpadas de xenônio). Isto se deve ao fato de as de mercú-

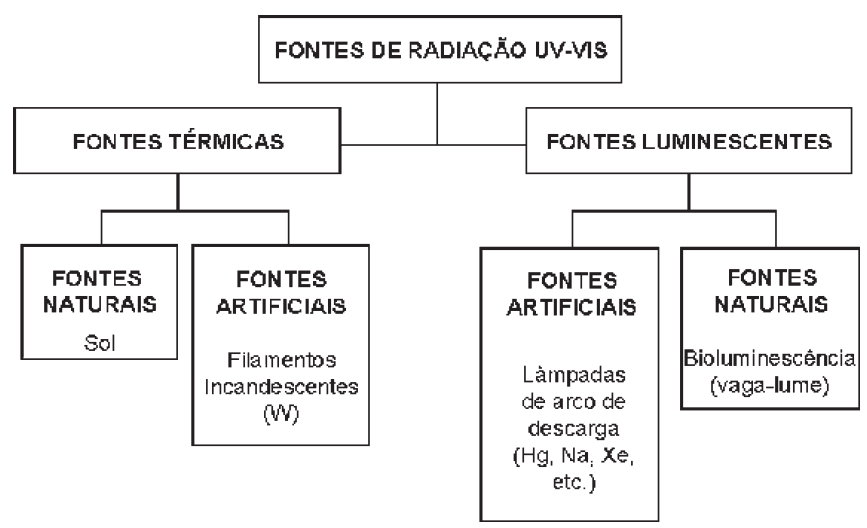

Figura 1. Classificação de fontes luminosas rio associarem a um relativo custo baixo e facilidade de funcionamento dois outros fatores, boas eficiências energética (proporção de potência elétrica convertida em radiação) e espectral (proporção de radiação emitida no $\mathrm{UV})^{9-11}$.

Nas lâmpadas de mercúrio por descarga em arco, a irradiação decorre do relaxamento luminescente de átomos de mercúrio excitados pelas colisões resultantes da corrente de elétrons e íons no arco formado entre os eletrodos montados no interior de um bulbo de quartzo, contendo, além de uma determinada quantidade do metal, um gás nobre - normalmente argônio ${ }^{11}$. O espectro de emissão depende fortemente da pressão dos gases no interior do bulbo havendo, por este motivo, a distinção entre lâmpadas de baixa, média e alta pressão. As propriedades dos três tipos de lâmpadas de mercúrio são resumidas na Tabela 1 .

As lâmpadas de baixa pressão são essencialmente monocromáticas com $\lambda=253 \mathrm{~nm}$, sendo que uma outra linha gerada em $189 \mathrm{~nm}$ é, em lâmpadas convencionais, filtrada pelas paredes do bulbo e pelo $\mathrm{O}_{2}$ atmosférico. Sua eficiência de conversão da potência em radiação UV é melhor do que as lâmpadas de média e alta pressão que, apesar de apresentarem potência nominal aproximadamente uma ordem de grandeza maior, convertem em luz UV somente cerca de $30 \%$ da potência elétrica consumida ${ }^{8}$. No entanto, para compensar a baixa intensidade luminosa das lâmpadas de baixa pressão, torna-se conveniente a construção de bulbos compridos, às vezes enrolados em forma de espiral, de maneira a se obter um maior fluxo luminoso total. Já as fontes de média e alta pressão apresentam um espectro de emissão caracterizado por linhas mais alargadas e um fundo contínuo que cobre toda a região $\mathrm{UV}$, inclusive a faixa do ultravioleta próximo (UV-A) e têm incursão na região visível, emitindo também uma grande quantidade de calor que, em equipamentos analíticos, pode ser, segundo exigido,

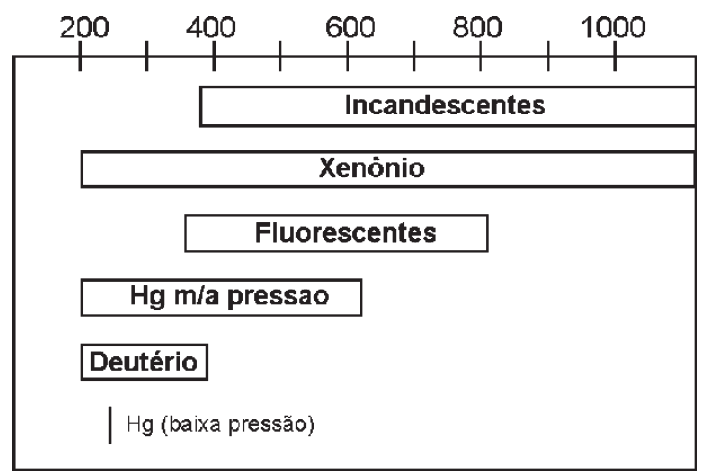

Figura 2. Domínio dos espectros de emissão dos principais tipos de lâmpadas ( $\lambda$ em $\mathrm{nm})$

Tabela 1. Características das lâmpadas de vapor de mercúrio

\begin{tabular}{|c|c|c|}
\hline Tipo de lâmpada & Espectro de emissão & Características \\
\hline Baixa Pressão $10^{-3}-10^{1}$ torr & $\begin{array}{l}\text { Duas bandas estritas em } 253,7 \mathrm{~nm}(80 \%) \\
\text { e } 189,9 \mathrm{~nm} \text {. Elevada taxa de conversão da } \\
\text { potência elétrica consumida em radiação UV }\end{array}$ & $\begin{array}{l}\text { - Tempo de vida relativamente longo } \\
\text { - Precisam de maior comprimento para proporcionar } \\
\text { aumento do fluxo radiante } \\
\text { - Temperatura típica: } 40^{\circ} \mathrm{C}\end{array}$ \\
\hline $\begin{array}{l}\text { Média Pressão } \sim 10^{3} \text { torr } \\
\text { Alta Pressão } \sim 8 \times 10^{4} \text { torr }\end{array}$ & $\begin{array}{l}\text { Apresentam várias bandas alargadas na região } \\
\text { UV, sendo os picos em } 365 \mathrm{~nm} \text { (média pressão) } \\
\text { e em } 436 \text { e } 546 \mathrm{~nm} \text { (alta pressão) os mais intensos. } \\
\text { Ocorre emissão não desprezível no visível (15\%*) } \\
\text { e no infravermelho (35\%*) }\end{array}$ & $\begin{array}{l}\text { - Tempo de vida inferior ao das de baixa pressão } \\
\text { - Elevado fluxo radiante, portanto podem ser compactas } \\
(10-40 \mathrm{~cm}) \\
\text { - Atingem temperaturas elevadas }\left(600-800{ }^{\circ} \mathrm{C}\right)\end{array}$ \\
\hline
\end{tabular}

*típico para lâmpadas de média pressão 
utilizado para aquecer as amostras tratadas. Ademais, as lâmpadas de baixa pressão operam em baixas temperaturas ${ }^{10}$ (tipicamente, $40{ }^{\circ} \mathrm{C}$ ) sendo que o metal no seu interior pode estar parcialmente condensado, ao passo que as de média e alta pressão alcançam valores de até 600$800{ }^{\circ} \mathrm{C}$ em que todo o mercúrio é completamente vaporizado. Por esta mesma razão, a quantidade de mercúrio no estado de vapor é determinada, no primeiro tipo de lâmpada, pela sua pressão parcial, isto é pela temperatura, enquanto nos demais modelos é definida pela quantidade de metal inserido no bulbo durante a manufatura que, portanto, deve ser cuidadosamente estabelecida.

Cabe registrar a possibilidade de se utilizar, como fontes de UV, lâmpadas de mercúrio (e respectivo reator) do tipo usado em larga escala para iluminação pública, facilmente disponíveis no comércio, a custo relativamente baixo, como sugerido antes por Aguiar $^{12}$ e depois por Cavicchioli e Gutz ${ }^{13}$. Para tanto, é suficiente remover (p.ex., com a ajuda de uma serra diamantada) o invólucro externo de vidro, cujas paredes internas são cobertas por material fluorescente, expondo o núcleo interno da lâmpada, constituído precisamente por uma lâmpada de mercúrio de média/alta pressão num invólucro de quartzo. Como todas as lâmpadas de mercúrio, estas também devem ser operadas com o suporte de um reator externo, geralmente uma impedância indutiva, que serve para compensar a característica voltagem-corrente negativa de condutores gasosos e manter um funcionamento estável (para mais informações, consultar Phillips ${ }^{10}$ ).

Na construção dos foto-reatores, os fatores-chave são: o melhor aproveitamento da radiação, com otimização de esquemas de reflexão e focalização, e a questão do controle da temperatura. A dissipação de calor pela lâmpada acesa é intensa e sua absorção pelas amostras pode acelerar o processo desejado. Todavia, a temperatura de ebulição pode ser rapidamente alcançada, dependendo da geometria, obrigando à adição de dispositivo de resfriamento como, por exemplo, uma ventoinha. Todavia, ventilação exagerada é indesejável, quer por desacelerar a reação, quer por prejudicar a emissão da lâmpada, já que a temperatura altera a largura das bandas e sua intensidade relativa. O mais apropriado é controlar a temperatura com um termostato (p.ex., ligando ou acelerando a ventoinha conforme necessário).

A geometria de reator mais comum (Figura 3a) é a que prevê uma fonte tubular em posição axial a um invólucro externo usado tanto para bloquear a radiação e refleti-la para o interior, quanto para guiar um fluxo de ar, originado pela ventoinha na base do dispositivo. A circulação de água de refrigeração ao redor dos recipientes da amostra constitui alternativa mais eficiente, mas mais difícil de implementar, para otimizar a troca de calor, vez que a temperatura e a emissão - do bulbo da lâmpada se mantém em nível mais elevado. Para tratamento em batelada, a amostra é geralmente colocada em um ou vários tubos de quartzo tampado(s), disposto(s) ao redor da fonte. Existem no mercado fotodigestores completos como os produzidos pela Metrohm, mas versões simplificadas podem ser construídas no próprio laboratório, como feito por Campos et al. ${ }^{14}$.

Já para equipamentos acoplados com/ ou integrados em sistemas de fluxo, a solução convencional - adotada nos equipamentos de mineralização para determinação de carbono total - é a de circular a amostra por uma bobina que envolve a lâmpada, sendo esta confeccionada em quartzo, já que a transparência do vidro cai rapidamente à medida que se adentra a região do UV (Figura 3b). Porém em certos setores da fotoquímica, vem se impondo o uso de serpentinas de $\mathrm{PTFE}^{15,16}$, com transparência superior à do vidro, mas inferior à do quartzo frente à radiação UV, compensada em parte por melhor aproveitamento da radiação em decorrência da reflexão múltipla da radiação no interior das paredes do tubo, que apresenta índice de refração próximo ou mesmo inferior ao da solução irradiada. A vantagem de se substituir quartzo por PTFE é evidente, pois assim
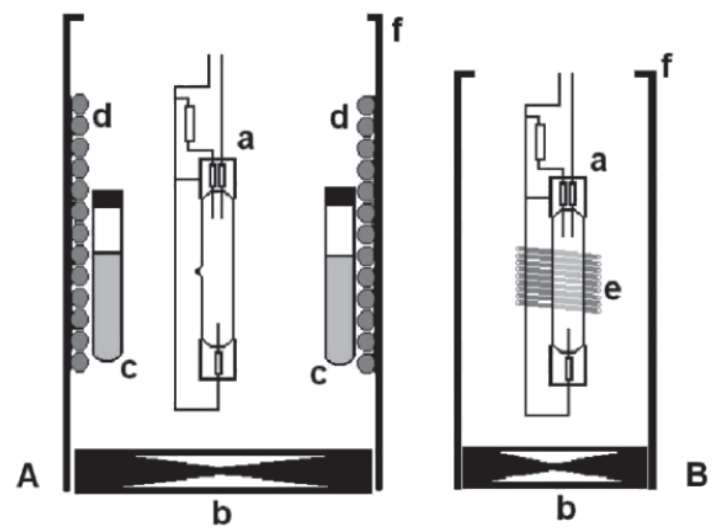

Figura 3. Exemplos de foto-reatores para decomposição em batch (A) e em fluxo (B). Lâmpada de médialalta pressão de $\mathrm{Hg}($ a); ventoinha (b); tubos de ensaio com amostra (c); sistema de resfriamento a água (d); bobina para a circulação da solução (e); paredes externas, espelhadas (f).

se eliminam os inconvenientes de custos mais elevados do material e dificuldade de manufatura, de menor flexibilidade na escolha da geometria da bobina e de fragilidade relacionada ao uso do quartzo ${ }^{16}$. Como exemplo de reatores em fluxo, aponta-se os sistemas de Achterberg e van den Berg ${ }^{17}$ e Cavicchioli e Gutz ${ }^{13}$.

Os mecanismos fotoquímicos de reação envolvem várias etapas que podem ser agrupadas em processos primários e secundários 9 . Nos processos primários, ocorre interação direta entre a radiação e espécies químicas presentes no meio, sejam elas próprias os alvos da reação, sejam outras substâncias adicionadas ou presentes naturalmente (como $\mathrm{O}_{2}$ dissolvido). O primeiro passo é sempre uma transição eletrônica, ou seja, a passagem para um estado excitado. A reatividade de espécies excitadas eletronicamente é, evidentemente, bem diferente daquela de moléculas no estado fundamental e, portanto, sua subseqüente evolução através de mecanismos proibidos às reações térmicas, as leva a formar produtos que seria impossível alcançar a partir da molécula no estado não excitado. As espécies excitadas $\left(\mathrm{AB}^{*}\right)$ logo evoluem de várias maneiras ${ }^{9}$ :

Dissociação

$\mathrm{AB}^{*} \rightarrow \mathrm{A}+\mathrm{B}$

Ionização

$\mathrm{AB}^{*} \rightarrow \mathrm{AB}^{+}+\mathrm{e}^{-}$

Reação com outras espécies

$\mathrm{AB}^{*}+\mathrm{E} \rightarrow \mathrm{ABE}$

$\mathrm{AB}^{*}+\mathrm{E} \rightarrow \mathrm{AE}+\mathrm{B}$

Isomerização

$\mathrm{AB}^{*} \rightarrow \mathrm{BA}$

Transferência de energia intra ou intermolecular $\mathrm{AB}^{*} \rightarrow \mathrm{AB}^{* *}$

$\mathrm{AB}^{*}+\mathrm{CD} \rightarrow \mathrm{AB}+\mathrm{CD}^{*}$

Luminescência (fluorescência ou fosforescência) $\mathrm{AB}^{*} \rightarrow \mathrm{AB}+\mathrm{hn}$

"Quenching"

$\mathrm{AB}^{*}+\mathrm{M} \rightarrow \mathrm{AB}$

(a energia é dissipada por $\mathrm{M}$ na forma de energia vibracional ou translacional) 
Os processos secundários são reações térmicas convencionais, que permitem converter espécies intermediárias geradas durante a primeira etapa e são fotoquímicos somente no sentido de que envolvem espécies formadas por efeito da radiação. Tais intermediários incluem espécies que são típicas das reações fotoquímicas como átomos e radicais livres e que apresentam uma reatividade elevada e peculiar, gerando reações em cadeia. Por exemplo ${ }^{17}$ :

$\mathrm{R}^{\bullet}+\mathrm{O}_{2} \rightarrow \mathrm{ROO}^{*}$

$\mathrm{ROO}^{\circ}+\mathrm{RH} \rightarrow \mathrm{ROOH}+\mathrm{R}^{\bullet}$

Neste contexto, destaca-se o papel do $\mathrm{O}_{2}$ e a formação de espécies altamente reativas como o oxigênio singlete $\left({ }^{1} \mathrm{O}_{2}\right)^{8,17}$ :

$\mathrm{S}^{*}+\mathrm{O}_{2} \rightarrow \mathrm{S}+{ }^{1} \mathrm{O}_{2}$

assim como o radical hidroxila, apontado como o maior responsável no processo de destruição de moléculas orgânicas":

$\mathrm{O}_{2}+\mathrm{hv} \rightarrow 2 \mathrm{O}$

$\mathrm{O}_{2}+\mathrm{O} \rightarrow \mathrm{O}_{3}$

$\mathrm{O}_{3}+\mathrm{H}_{2} \mathrm{O} \rightarrow \mathrm{H}_{2} \mathrm{O}_{2}+\mathrm{O}_{2}$

$\mathrm{H}_{2} \mathrm{O}_{2}+\mathrm{hv} \rightarrow \mathrm{HO}^{*}$

ou, ainda, o radical superóxido ${ }^{8-17}$ :

$\mathrm{H}_{2} \mathrm{O}_{2} \leftrightarrows \mathrm{HO}_{2}^{-}+\mathrm{H}^{+}$

$\mathrm{HO}_{2}^{-}+\mathrm{O}_{3} \rightarrow \mathrm{O}_{3}^{-\cdot}+\mathrm{HO}_{2}^{\cdot} \leftrightarrows \mathrm{O}_{2}^{-\bullet}+\mathrm{H}^{+}$

Observe-se a formação intermediária de oxidantes característicos como $\mathrm{H}_{2} \mathrm{O}_{2}$ e $\mathrm{O}_{3}$.

Foram evidenciados diversos esquemas de ação envolvendo HO', seja de propagação radicalar ${ }^{17}$ :

$\mathrm{HO}^{\bullet}+\mathrm{RH} \rightarrow \mathrm{R}^{\bullet}+\mathrm{H}_{2} \mathrm{O}$

ou ainda de adição ou substituição, como no caso do fenol que, durante sua oxidação é gradativamente convertido em hidroquinona, catecol e pirogalol, antes de sofrer abertura do anel aromático e descarboxilação ${ }^{18}$.

\section{DECOMPOSIÇÃO DE MATRIZES ORGÂNICAS POR IRRADIAÇÃO UV}

Atualmente, na área da análise elementar, as expressões pré-tratamento ou preparo de amostra viraram sinônimos de abertura ("digestion"/digestão) de amostra; um termo que indica os processos voltados à solubilização de amostras sólidas e/ou à eliminação dos interferentes, via de regra orgânicos, em consideração às exigências da técnica de determinação usada, mediante a decomposição, geralmente em temperaturas elevadas, da matriz onde se encontra incorporado o elemento alvo. Esta sobreposição de termos explica-se pelo fato da abertura de amostra representar, muitas vezes e, especialmente no caso de detecção por técnicas de espectroscopia atômica, a principal, quando não a única e mais complexa etapa preliminar da análise.

Feita esta ressalva e passando a considerar os processos de abertura de amostra, cabe lembrar que estes podem ser classificados, com base no tipo de energia fornecida ao sistema, em métodos térmicos (transmissão de calor), radiantes (incidência de onda eletromagnéticas ou mecânicas) e eletroquímicos (Tabela 2). Os chamados métodos térmicos clássicos representaram, no passado, a abordagem usual para a abertura de amostras. Embora sejam ainda amplamente utilizados, as metodologias baseadas em fornos de microondas vieram a ser apontadas cada vez mais como a solução mais conveniente e versátil para a etapa de preparo da amostra. Trata-se sempre de métodos térmicos, pois neles somente muda a modalidade de aquecimento da amostra e não o princípio de utilizar calor para a ruptura das ligações químicas nos substratos a serem decompostos. Ao usar as microondas, não há necessidade de aquecer o forno em si, pois o incremento de temperatura se dá, de modo rápido, eficaz, uniforme e reprodutível, pela migração de íons e rotação de dipolos (permanentes ou induzidos) no meio reacional, por absorção da radiação eletromagnética incidente, cabendo assinalar que a própria água absorve radiação nesta região espectral, pelo segundo processo apon$\operatorname{tado}^{19}$.

Tabela 2. Classificação das técnicas de abertura de amostra

\begin{tabular}{ll}
\hline Métodos térmicos & $\begin{array}{l}\text { via úmida } \\
\text { via seca } \\
\text { fusão }\end{array}$ \\
& microondas \\
& ultravioleta \\
infravermelho & ultra-som \\
Métodos radiantes & raios X
\end{tabular}

Eletroquímicos

Técnicas de mecanização da abertura em fornos de microondas e as modalidades para sua execução em regime de fluxo têm sido investigadas com interesse, devido à importância de simplificar e acelerar o procedimento, muitas vezes demorado, quando praticado em batelada. Burguera e Burguera ${ }^{20}$ e, depois, Arruda e Santelli ${ }^{2}$ revisaram as tentativas realizadas até então para integrar fornos de microondas em sistemas de fluxo, destacando a dificuldade de lidar com a excessiva pressão gasosa gerada pela decomposição de matéria orgânica, na presença de ácidos concentrados e oxidantes fortes. Processos de abertura de amostra em fornos de microondas não são livres de riscos de explosão devido aos rápidos ciclos de aquecimento e subseqüente pressurização, decorrente da formação de produtos gasosos, especialmente na presença de material orgânico. Estes eventos, que às vezes são difíceis de serem previstos ou - quando ocorrem - mantidos sob controle, tornam-se ainda mais insidiosos pelo fato da amostra, quase invariavelmente ser misturada a substâncias agressivas, normalmente ácidos e oxidantes, em alguns casos extremamente lesivos (p.e. $\mathrm{HF}, \mathrm{HClO}_{4}$ ). De qualquer maneira, equipamentos e sistemas para digestão em linha, apesar das dificuldades apresentadas, são já disponíveis comercialmente ${ }^{2}$ e a pesquisa nesta área consolidou-se nos últimos anos, produzindo várias soluções ao problema (inclusive, utilizando simples sistemas artesanais em fluxo ${ }^{21}$ ).

Outras abordagens para digestão de amostras, tais como a decomposição assistida por ultra-som ${ }^{22}$ e infravermelho ${ }^{23}$, vêm recebendo crescente interesse por parte da comunidade científica, em parte como resultado de uma maior e generalizada atenção para as técnicas de pré-tratamento e, por outro, devido à busca de alternativas às metodologias térmicas que podem vir a ser pouco viáveis, por conta da dificuldade de serem implementadas em fluxo e da necessidade de reagentes adicionais. Deve-se ressalvar que a adição de ácidos e oxidantes, além de incorporar o risco de contaminação especialmente nas determinações de elementos de tipo traço, pode tornar o meio analítico incompatível com a técnica de detecção. Por exemplo, na voltametria, um meio extremamente ácido significa antecipação da descarga de hidrogênio (quando não, a da oxidação do $\mathrm{Hg}$, ao 
usar-se eletrodos deste metal) causando, possivelmente, a elevação da linha-base, enquanto o $\mathrm{H}_{2} \mathrm{O}_{2}$ - também amplamente utilizado em fornos de microondas - interfere no registro do voltamograma em potenciais negativos. A digestão eletroquímica pode ser abordada de maneira semelhante aos métodos ilustrados para tratamento de efluentes por Rajeshwar e Ibañez ${ }^{24}$, mas é raro encontrar metodologias de decomposição eletroquímica aplicadas ao tratamento de amostra (ver, p. ex., Brainina et al. ${ }^{25}$ ).

O uso da irradiação ultravioleta para a decomposição de matrizes orgânicas surgiu entre a década de 1960 e 1970. Golimowski, autor de ampla revisão bibliográfica sobre o assunto ${ }^{8}$, aponta que a idéia pioneira de se valer deste meio para promover a decomposição de matéria orgânica em água de mar visando a determinação de elementos e compostos inorgânicos, se encontra num trabalho de $1966^{26}$. Nos anos seguintes, essa metodologia foi aplicada com grande freqüência em amostras de águas naturais (rios, lagos, mar, chuva, neve, orvalho) para a determinação voltamétrica do conteúdo total de metais, havendo neste campo contribuições de destaque pelo grupo de Nürnberg e Valenta, na Alemanha ${ }^{27}$, os mesmos que desenvolveram um dos primeiros protótipos de fotodigestor descritos na literatura, acoplado a um analisador voltamétrico e automatizado ${ }^{28}$.

A técnica tornou-se, assim, quase padrão para a análise voltamétrica de íons de metais como $\mathrm{Zn}, \mathrm{Cd}, \mathrm{Pb}_{\text {e }} \mathrm{Cu}^{29}$ e não só em águas de origem ambiental, bem como em efluentes domésticos e urbanos ${ }^{30}$. Interessantes investigações sistemáticas sobre o uso da fotodegradação por radiação ultravioleta para a destruição de matéria orgânica visando a determinação do conteúdo total de metais pesados foram realizadas utilizando soluções modelo de EDTA, acido pícrico e Triton X-100 em mistura ${ }^{31}$ ou de ácidos húmicos comerciais ${ }^{14,17,32}$. No primeiro trabalho, utilizou-se soluções aciduladas com $\mathrm{HCl}(\mathrm{pH}$ 2) de íons de $\mathrm{Zn}$, $\mathrm{Pb}$ e $\mathrm{Cu}\left(10 \mu \mathrm{g} \mathrm{L}^{-1}\right), \mathrm{Cd}$, Ni e Co $\left(2 \mu \mathrm{g} \mathrm{L}^{-1}\right)$, EDTA, ácido pícrico e Triton X-100 (10 $\left.\mathrm{mg} \mathrm{L}^{-1}\right)$ visando simular os diferentes efeitos de potenciais interferentes, ou seja complexação estável de metais (EDTA), atividade eletroquímica no mesmo potencial que os íons metálicos hidratados (ácido pícrico) e passivação do eletrodo de trabalho (Triton $\mathrm{X}-100$ ), na presença de metais pesados eletroativos, em baixas concentrações. Comprovou-se a completa recuperação do sinal de $\mathrm{Zn}$, $\mathrm{Cd}, \mathrm{Pb}$ e $\mathrm{Cu}$ com 30 min de irradiação com lâmpada de mercúrio de alta pressão de $500 \mathrm{~W}$ a $65^{\circ} \mathrm{C}$ sem $\mathrm{H}_{2} \mathrm{O}_{2}$. Para Ni e Co foram necessárias $2,5 \mathrm{~h}$ à mesma temperatura. $\mathrm{O}$ método foi também testado com sucesso em soluções reais de água de rio e lago e efluentes urbanos com irradiações de $1 \mathrm{~h} \mathrm{a} 60{ }^{\circ} \mathrm{C}$ com $\mathrm{H}_{2} \mathrm{O}_{2}$. Já com resíduos da indústria galvanoplástica e de usinas termoelétricas a carvão, prolongados tratamentos não foram suficientes para a completa recuperação dos metais. Além do efeito benéfico de temperatura elevada (até $90{ }^{\circ} \mathrm{C}$ ) e do $\mathrm{H}_{2} \mathrm{O}_{2}$, os autores observaram, interessantemente, a interferência do íon nitrito, formado fotoquimicamente, no sinal do $\mathrm{Zn}$. Cabe porém notar que, do ponto de vista meramente analítico, o que importa não é, necessariamente, a completa mineralização dos compostos orgânicos, mas sim a perda da capacidade complexante perante os íons metálicos ou, pelo menos, a formação de adutos lábeis quando se visa a determinação eletroanalítica.

Nos trabalhos de Achterberg e van den Berg ${ }^{17}$ e Campos et al. ${ }^{14}$, a destruição de ácidos húmicos comerciais foi acompanhada mediante detecção fluorimétrica, portanto também sem confirmação da completa mineralização. Porém, em ambos os casos, os resultados foram subseqüentemente sustentados por ensaios em amostras reais. Achterberg e van den Berg ${ }^{17}$ realizaram digestão de amostras sintéticas de ácidos húmicos em água de mar e água bidestilada, observando que, nesta última, o substrato se decompõe mais facilmente. Compararam também processos em batelada e em fluxo chegando a afirmar a superioridade da segunda abordagem, atribuindo-a à mais elevada razão superfície/volume da serpentina utilizada para a digestão em flu- xo, em relação aos tubos de ensaio utilizados no digestor tipo "batch". Amostras reais de mar foram satisfatoriamente analisadas após fototratamento em fluxo para a determinação voltamétrica de íons de $\mathrm{Cu}$, $\mathrm{Ni}$ e $\mathrm{Cu}$, com tempos de irradiação de $150-175$ s a $70{ }^{\circ} \mathrm{C}$ e em presença de $\mathrm{H}_{2} \mathrm{O}_{2}$. Campos et al. ${ }^{14}$ também testaram soluções de ácidos húmicos comerciais nas concentrações de 4, 6, 8 e $10 \mathrm{mg} \mathrm{C} \mathrm{L}^{-1}$ conseguindo com todas uma concentração $<0,01 \mathrm{mg} \mathrm{C} \mathrm{L}^{-1}$ após irradiações de 30 , $60,>60,>60 \mathrm{~min}$, respectivamente. $\mathrm{Cu}$ foi determinado voltametricamente em amostras reais de água doce e salgada, após fotodigestão de $3 \mathrm{~h}$. Neste trabalho utilizou-se um dispositivo artesanal provido de lâmpada de alta pressão de $125 \mathrm{~W}$, circundada por tubos de ensaio de quartzo com capacidade de 42,5 mL mantidos, durante os experimentos relatados, a $70{ }^{\circ} \mathrm{C}$.

Entretanto, nos casos mais drásticos, requer-se um tempo de exposição elevado e adição de $\mathrm{H}_{2} \mathrm{O}_{2}$ para possibilitar completa recuperação dos sinais analíticos. $\mathrm{O}$ uso de peróxido, embora muito valioso, nem sempre é viável pois, conforme já mencionado, é eletroativo e sua presença na célula eletroquímica pode mascarar o sinal analíti$\mathrm{Co}^{33}$. Outros oxidantes fortes como $\mathrm{MnO}_{4}^{-}, \mathrm{Cr}_{2} \mathrm{O}_{7}^{2-} \mathrm{e} \mathrm{S}_{2} \mathrm{O}_{8}^{2-}$ foram sugeridos pois permitem estender o uso da fotoirradiação para a digestão de matrizes mais complexas do que as águas naturais ${ }^{8}$. São relatadas determinações de metais pesados e metalóides em matrizes como vinho, extratos de solo, efluentes industriais, extratos de couro, bebidas alimentares e fluidos biológicos ${ }^{8}$, registrando-se várias modalidades no tocante ao tipo e à potência da lâmpada, à presença de aditivos e temperatura. Devido à diferença nestes parâmetros, mesmo com matrizes do mesmo tipo, a duração da exposição varia de poucos minutos até várias horas. Repita-se que a técnica de detecção determina o rigor com que deve ser conduzida a digestão, sendo que a espectroscopia atômica, via de regra, é menos afetada pela presença de material parcialmente degradado que a voltametria.

Uma outra área de aplicação é representada por sistema de determinação total, principalmente de $\mathrm{C}, \mathrm{N}$ e $\mathrm{P}$ orgânicos, mas também $\mathrm{S}$ e halogênios ${ }^{8,34}$. Via de regra, estes processos requerem a presença de oxidantes para garantir a conversão quantitativa. Surgiram, neste setor, os primeiros exemplos de dispositivos automáticos com a irradiação em fluxo, uma das tendências mais marcantes do emprego da digestão por irradiação UV em anos recentes. Acheterberg e van den Berg $^{17}$ foram os primeiros que descreveram um sistema de digestão UV em linha, compreendendo lâmpada de mercúrio de média pressão de $100 \mathrm{~W}$ e uma serpentina de sílica envolvendo a fonte, e comparam seu desempenho com aquele de um digestor convencional.

$\mathrm{O}$ ingresso de esquemas de fotodegradação em fluxo e sua comprovada eficiência para águas naturais contendo substâncias húmicas impulsionou o desenvolvimento de sistemas integrados para análise de amostras reais, parcial ou completamente automatizados, para a realização de campanhas de monitoramento de curto ou longo alcance de metais pesados ${ }^{35}$.

Uma outra tendência que se desenvolveu a partir do renovado interesse por sistemas de irradiação em fluxo, foi o acoplamento com etapas de separação por cromatografia líquida. Buldini et al. ${ }^{36}$ propuseram um procedimento para determinar $\mathrm{Cl}, \mathrm{Br}, \mathrm{P}$ (como $\mathrm{PO}_{4}^{3-}$ ), $\mathrm{S}$ (como $\mathrm{SO}_{4}{ }^{2-}$ ) $\mathrm{Pb}, \mathrm{Cd}, \mathrm{Fe}, \mathrm{Cu}, \mathrm{Ni}, \mathrm{Zn}$ e Co totais em folhas, por cromatografia de íons após digestão UV (lâmpada de $500 \mathrm{~W}, 85^{\circ} \mathrm{C}$ ). Tsalev et al. ${ }^{37,38}$, Ebdon et al. ${ }^{39}$, Falter e Schöler ${ }^{40}$ e Zhang et al. ${ }^{41}$ apresentaram métodos em fluxo para estudar e quantificar a especiação de compostos orgânicos de As (em urina ${ }^{37}$ ou soro huma$\mathrm{no}^{41}$ ), $\mathrm{Sn}$ (em urina ${ }^{38}$ ou águas naturais ${ }^{39}$ ), $\mathrm{Hg}^{40}$ (amostras sintéticas) através de sistemas integrados de separação em colunas HPLC, destruição em digestor UV, geração de hidretos (As e Sn) ou geração de vapor frio $(\mathrm{Hg})$ e detecção por AAS.

Como se pode ver, se nos primeiros anos, a decomposição por UV realizou uma associação muito profícua com as técnicas 
voltamétricas, porém em aplicações restritas ao pré-tratamento de amostras de água, o renovado interesse registrado na última década fez com que fosse levada em conta como técnica de abertura de uma gama mais ampla de amostras e para detectores não eletroquímicos. Para tanto, as pesquisas visaram encontrar métodos de incrementar sua eficácia, de preferência sem a necessidade de oxidantes adicionais como $\mathrm{H}_{2} \mathrm{O}_{2}$.

Yokoi et al. ${ }^{32}$ recorreram a um novo modelo de lâmpada presente no mercado, de baixa pressão, de elevada potência e mostraram que sua eficácia é nitidamente melhor do que as lâmpadas de baixa pressão/baixa potência e média pressão/igual potência na foto-decomposição de várias substâncias orgânicas, inclusive ácidos húmicos. Esta pesquisa indicou também uma melhor performance quando a fotodegradação é conduzida em meio ácido utilizando-se esta lâmpada, ao menos para os compostos tratados.

Uma contribuição mais recente ${ }^{42}$ destaca-se como proposta pioneira de realizar a abertura de amostra por irradiação simultânea de ultravioleta e microondas. Neste trabalho, uma ampola de quartzo contendo cádmio, cujo vapor produz emissão monocromática em $\lambda=228 \mathrm{~nm}$, é posicionada no fundo de um tubo de ensaio de quartzo juntamente com a solução ou suspensão a ser tratada e este conjunto é inserido na cavidade de um forno de microondas. A fonte de radiação UV é desprovida de eletrodo pois as próprias microondas desempenham o duplo papel de fornecer energia à amostra e de iniciar e manter o processo de excitação dos átomos de $\mathrm{Cd}$, responsável pela emissão no UV. Esta nova abordagem foi testada com sucesso em uma amostra de material certificado de leite desnatado, possibilitando $99 \%$ de decomposição do material orgânico em 30 min de tratamento.

Sempre no sentido de aprimorar a foto-decomposição de matéria orgânica, Cavicchioli e Gutz ${ }^{13}$ apresentaram a proposta de se valer do efeito fotocatalítico de partículas de $\mathrm{TiO}_{2}$ em suspensão. $\mathrm{O}$ emprego de fotocatalisadores em fase heterogênea para acelerar processos de degradação de substâncias orgânicas é bem conhecido há cerca de duas décadas, mas foi sempre relacionado ao tratamento de efluentes e não ao pré-tratamento de $\operatorname{amostras}^{43}$. No estudo citado, comprovou-se - ainda que para sistema-modelo e número limitado de amostras reais - a conveniência desta aproximação que proporciona um significativo aumento da velocidade de decomposição do substrato, além de deslocar a faixa de aproveitamento da radiação UV para regiões próximas ao visível, pois o $\mathrm{TiO}_{2}$-anatase, o principal componente do Degussa P25 comercial, absorve radiação UV a partir de $384 \mathrm{~nm}$. É claro que o uso de um material em suspensão como o $\mathrm{TiO}_{2}$, que confere à solução um aspecto leitoso, que não causa problema para sensores eletroanalíticos, especialmente quando renováveis como os eletrodos de gota de mercúrio, é inviável para detectores espectrofotométricos. Entretanto, esta restrição poderia ser superada com a imobilização do semicondutor nas paredes do foto-reator, uma possibilidade experimentada em outras áreas mas ainda não implementada neste tipo de aplicação analítica.

Para encerrar esta seção, ainda em relação à fotocatálise na presença de $\mathrm{TiO}_{2}$, é interessante mencionar a idéia de se utilizar este princípio para o pós-tratamento de amostras, ou seja para tratar resíduos de compostos orgânicos tóxicos empregados em procedimentos analíticos, depois da detecção e antes do descarte ${ }^{44}$. Novamente, o atrativo do uso de um procedimento de foto-decomposição está na possibilidade de integrar esta etapa adicional num esquema de análise em fluxo, eventualmente automatizado.

\section{REAÇÕES DE CONVERSÃO FOTOQUÍMICA NO PREPARO DE AMOSTRA}

Embora, no senso comum, o uso do UV no preparo de amostra esteja associado à decomposição de matéria orgânica para a determi- nação de compostos ou elementos inorgânicos, existe também a possibilidade de se valer de reações fotoquímicas para transformações prévias dos analitos, em procedimentos que, portanto, não se configuram como processos de destruição ou degradação de moléculas. Há quem, em relação a esta abordagem, fale em fotoquímica analítica ou análise fotoquímica ${ }^{45}$. No entanto, cumpre realçar que as aplicações em química orgânica prevalecem nitidamente sobre métodos de análise de espécies inorgânicas, sendo que o campo talvez mais fecundo em contribuições é o da derivatização de compostos orgânicos após separação cromatográfica em reatores denominados de póscoluna, visando detecção fluorimétrica ${ }^{16}$.

Alguns exemplos significativos de métodos analíticos que incluem etapas fotoquímicas são resumidos na Tabela 3. Ampliando o enfoque central deste artigo - a análise inorgânica - a tabela inclui casos de métodos visando a determinação de analitos orgânicos, em procedimentos envolvendo reações redox de elementos ou compostos inorgânicos.

Veja-se, para começar, o caso do ferro. Nos métodos apresentados, aproveita-se a redução de $\mathrm{Fe}(\mathrm{III})$ para $\mathrm{Fe}(\mathrm{II})$, freqüentemente com determinação de $\mathrm{Fe}$ (II) por espectrofotometria de absorção molecular no visível em $510 \mathrm{~nm}$, após formação do complexo Fe(II)-fenantrolina. O esquema é utilizado não somente com a finalidade de determinar o conteúdo total $\mathrm{Fe}(\mathrm{II})+\mathrm{Fe}(\mathrm{III})^{46}$, mas também na quantificação de ácidos orgânicos oxidáveis que formam complexos foto-sensíveis com o $\mathrm{Fe}(\mathrm{III})$, p.ex. ácido cítrico ${ }^{47}$ e ácido oxálico ${ }^{48}$, sendo que a espécie oxidante é o $\mathrm{Fe}(\mathrm{III})$ e a redutora o composto orgânico.

Neste tipo de reações, acredita-se que ocorra a transferência intramolecular de um elétron do anion orgânico para o Fe(III) ligado ao grupo ácido, com subseqüente descarboxilação ${ }^{46}$.

$-\mathrm{CO}-\mathrm{O}-\mathrm{Fe}^{3+}+\mathrm{hv} \rightarrow \mathrm{CO}_{2}+\mathrm{Fe}(\mathrm{II})$

Segundo Luque-Pérez et $a l .{ }^{47}$, a reação é bastante rápida (30 s) e parece pouco afetada pela temperatura.

$\mathrm{O}$ método para determinação de $\mathrm{F}^{-}$ou $\mathrm{PO}_{4}^{3-}$, proposto por PérezRuiz et $a .^{7}{ }^{7}$, também é baseado no sistema redox Fe(III)-ligante orgânico. Nesta aproximação, no entanto, aproveita-se o efeito inibidor dos analitos e sua concentração é medida indiretamente, comparando-se o sinal fluorimétrico de uma oportuna substância orgânica oxidável fotoquimicamente por Fe(III) (o alaranjado de acridina) num branco - onde não há nenhum efeito inibidor - e em amostras contendo $\mathrm{F}^{-}$ou $\mathrm{PO}_{4}^{3-}$.

O mesmo princípio da ação inibidora do analito pode ser explorado utilizando-se o sistema $\mathrm{I}_{2} /$ EDTA. No trabalho de SánchezPedreño et al. ${ }^{49}, \mathrm{Hg}$ (II) é detectado aproveitando-se o retardamento provocado por sua presença na foto-oxidação do EDTA por parte do $\mathrm{I}_{2}$. Este é um exemplo de determinação cinética, pois o analito atua na velocidade de reação e o parâmetro monitorado na quantificação é o tempo requerido para o completo consumo de $\mathrm{I}_{2}$ (determinado amperometricamente).

O mecanismo de reação $0^{50}$ prevê a ruptura homolítica da ligação I-I por ação da radiação:

$\mathrm{I}_{2}+\mathrm{hv} \rightarrow 2 \mathrm{I}^{\cdot}$

seguida por reações em cadeia que levam à descarboxilação do EDTA:

$\mathrm{R}_{1} \mathrm{R}_{2} \mathrm{~N}-\mathrm{CH}_{2} \mathrm{COO}^{-}+\mathrm{I}^{-} \rightarrow \mathrm{R}_{1} \mathrm{R}_{2} \mathrm{~N}_{-} \mathrm{CH}_{2}^{-}+\mathrm{I}^{-}+\mathrm{CO}_{2}$
$\mathrm{R}_{1} \mathrm{R}_{2} \mathrm{~N}_{-} \mathrm{CH}_{2}^{-}+\mathrm{I}_{2} \rightarrow \mathrm{R}_{1} \mathrm{R}_{2} \mathrm{~N}^{-} \mathrm{CH}_{2} \mathrm{I}+\mathrm{I}^{-}$
onde: $\mathrm{R}_{1}=\mathrm{CH}_{2}-\mathrm{COO}^{-}$e $\mathrm{R}_{2}=\mathrm{C}_{2} \mathrm{H}_{4}-\mathrm{N}\left(\mathrm{CH}_{2} \mathrm{COO}^{-}\right)_{2}$

Para este método, foi relatado um intervalo de aplicabilidade entre 0,4 e $6 \mathrm{ng} \mathrm{mL} \mathrm{m}^{-1}$ (ppb) de $\mathrm{Hg}(\mathrm{II})$. Neste, como em outros procedimen- 
Tabela 3. Exemplos de métodos analíticos incluindo etapas fotoquímicas

\begin{tabular}{|c|c|c|c|}
\hline Espécie foto-sensível & Analito & Observações & Ref. \\
\hline $\mathrm{Fe}(\mathrm{II})$ & $\mathrm{Fe}(\mathrm{II}) / \mathrm{Fe}(\mathrm{III})$ & $\begin{array}{l}\mathrm{Fe}(\mathrm{OH})^{2+}+\mathrm{h} v \rightarrow \mathrm{Fe}^{2+}+\mathrm{HO}^{\bullet} \\
2 \mathrm{HO}^{\bullet} \rightarrow \mathrm{H}_{2} \mathrm{O}+1 / 2 \mathrm{O}_{2} \\
\text { Detecção espectrofotométrica de } \mathrm{Fe}(\mathrm{II}) \text {-fenantrolina } \\
\text { Método em fluxo }\end{array}$ & 46 \\
\hline Ácido cítrico-Fe(II) & Ácido cítrico & $\begin{array}{l}\mathrm{Fe}(\mathrm{III})+\text { a. cítrico } \\
\text { red } \\
\text { Detecção espectrofotométrica de } \mathrm{Fe}(\mathrm{II}) \text {-fenantrolina } \\
\text { Método em fluxo }\end{array}$ & 47 \\
\hline $\mathrm{Fe}\left(\mathrm{C}_{2} \mathrm{O}_{4}\right)^{+}$ & $\mathrm{C}_{2} \mathrm{O}_{4}^{2-}$ & $\begin{array}{l}\mathrm{Fe}\left(\mathrm{C}_{2} \mathrm{O}_{4}\right)_{2}^{+}+\mathrm{h} v \rightarrow 2 \mathrm{Fe}^{2+}+2 \mathrm{CO}_{2}+\mathrm{C}_{2} \mathrm{O}_{4}^{2-} \\
\text { Método em fluxo }\end{array}$ & 48 \\
\hline Fe-org & $\mathrm{F}^{-}$ou $\mathrm{PO}_{4}^{3-}$ & $\begin{array}{l}\mathrm{Fe}(\mathrm{III})+\mathrm{Org}_{\text {red }}+\mathrm{h} v \rightarrow \mathrm{Fe}(\mathrm{II})+\mathrm{Org}_{\mathrm{ox}} \\
\text { Org=alarajado de acridina } \\
\text { Determinação fluorimétrica do alaranjado de acridina } \\
\text { A determinação de } \mathrm{F}^{-} \text {ou } \mathrm{PO}_{4}^{3-} \text { é baseada em seu efeito cinético (inibidor) } \\
\text { na foto-oxidação de Org por } \mathrm{Fe}(\mathrm{III}) \\
\text { Método em fluxo }\end{array}$ & 7 \\
\hline $\mathrm{I}_{2}$ & $\mathrm{NO}_{2}^{-}$ & $\begin{array}{l}\mathrm{I}_{2}+\text { hv } \rightarrow 2 \mathrm{I} \\
\mathrm{I}^{+} \text {EDTA } \\
\text { Detecção amperométrica de } \mathrm{I}^{-} \\
\text {A determinação de } \mathrm{NO}_{2}^{-} \text {é baseada no efeito cinético (inibidor) de } \mathrm{NO}_{2}^{-} \\
\text {na foto-oxidação de EDTA por } \mathrm{I}_{2} \\
\text { Método em fluxo }\end{array}$ & 50 \\
\hline $\mathrm{I}_{2}$ & $\mathrm{Hg}(\mathrm{II})$ & $\begin{array}{l}\mathrm{I}_{2}+\text { hv } \rightarrow 2 \mathrm{I} \\
\mathrm{I}^{+} \mathrm{EDTA}_{\text {red }} \rightarrow \mathrm{I}^{-}+\mathrm{EDTA}_{\text {ox }} \\
\text { Detecção amperométrica de } \mathrm{I}^{-} \\
\text {A determinação de } \mathrm{Hg}(\mathrm{II}) \text { é baseada no efeito cinético (inibidor) de metal } \\
\text { na foto-oxidação de EDTA por } \mathrm{I}_{2} \\
\text { Método em "batch" com irradiação in-situ }\end{array}$ & 49 \\
\hline $\mathrm{NO}_{3}^{-}$ & $\mathrm{NO}_{3}^{-}$ & $\begin{array}{l}\text { Detecção- por quimiluminescência: } \mathrm{NO}_{3}^{-} \text {forma, em meio ácido, } \\
\text { peroxonitrito responsável pela oxidação de luminol } \\
\text { Método em fluxo }\end{array}$ & 53 \\
\hline $\mathrm{NO}_{3}^{-}$ & $\mathrm{NO}_{3}^{-}$ & $\begin{array}{l}2 \mathrm{NO}_{3}^{-}+2 \mathrm{I}^{-}+\mathrm{hv} \rightarrow 2 \mathrm{NO}_{2}^{-}+\mathrm{I}_{2} \\
\text { Determinação biamperométrica de } \mathrm{I}_{3}^{-} \\
\text {Método em fluxo }\end{array}$ & 54 \\
\hline
\end{tabular}

tos realizados em "batch", a irradiação da amostra ocorre in situ, ou seja, na própria célula amperométrica, ao passo que nos sistemas em fluxo a incidência da radiação se dá num reator colocado logo antes do detector. É o caso do método em fluxo proposto por Liu e Liu ${ }^{50}$ para a determinação de nitrito na presença do sistema $\mathrm{I}_{2} /$ EDTA. No trabalho apresenta-se um sistema em fluxo integrando um reator para a redução fotoquímica de $\mathrm{I}_{2}$ e uma célula para a determinação amperométrica (de $\mathrm{I}_{2}$ ). Novamente, o $\mathrm{NO}_{2}^{-}$atua como inibidor da reação fotoquímica e é quantificado em função da quantidade de $\mathrm{I}_{2}$ residual que alcança o detector.

Contudo, entre todas as aplicações de reações fotoquímicas em análise inorgânica, a mais comum é a conversão de nitrato para nitrito. Este processo, bem conhecido há várias décadas, ocorre segundo este mecanismo ${ }^{51}$ :

$\mathrm{NO}_{3}^{-}+\mathrm{hv} \leftrightarrows \mathrm{NO}_{2}^{-}+\mathrm{O}$

$\mathrm{H}_{2} \mathrm{O}+\mathrm{O} \leftrightarrows \mathrm{H}_{2} \mathrm{O}_{2}$

Cultrera e Ferrari ${ }^{51}$ relatam que o íon $\mathrm{NH}_{4}^{+}$também é sujeito à foto-conversão para nitrito, no entanto não foram encontrados na literatura exemplos específicos de aplicações analíticas deste princípio.
Gil Torró et al. ${ }^{52}$ utilizam um reator de PTFE ao redor de uma lâmpada de mercúrio de baixa pressão para reduzir nitrato em fluxo, realizando a detecção de nitrito por biamperometria. Assim, conseguiram eliminar o uso de colunas redutoras de $\mathrm{Cu}-\mathrm{Cd}$, freqüente na determinação de nitrato como nitrito e aplicaram o método com sucesso à análise de $\mathrm{NO}_{3}^{-}$em vários tipos de amostras reais.

Em outro método ${ }^{53}$, o íon nitrato pode também ser determinado por quimioluminescência após formação fotoquímica de espécies oxidantes, como peroxinitrito, que causam a oxidação de luminol, responsável pelo efeito luminescente.

Ainda cabe registrar um método ${ }^{54}$ para a determinação de $\mathrm{NO}_{3}^{-}$, $\mathrm{NO}_{2}^{-}$e $\mathrm{N}_{\text {org total }}$ utilizando a radiação $\mathrm{UV}$, tanto para destruir o substrato orgânico numa primeira etapa, como para reduzir o nitrato (inclusive a porção que se forma após a irradiação) e realizar a determinação sequiencial dos três compostos na forma de nitrito, mediante a reação de Griess. No sistema em fluxo, um conjunto de válvulas admite seletivamente as amostras às etapas prévias, o que permite quantificar separadamente as três espécies.

Para encerrar esta seção, é oportuno ainda mencionar dois exemplos de pré-tratamento fotoquímico, envolvendo a determinação e a especiação de íons metálicos. Mattsson et al. ${ }^{55}$ apresentaram um 
método, em "batch", para a determinação de Se total em amostras de água por voltametria de "stripping" catódico. Esta técnica requer a presença de Se no estado de oxidação IV para ser depositado/préconcentrado no eletrodo de mercúrio como $\mathrm{Cu}_{2} \mathrm{Se}$. Assim, demonstrou-se ser possível reduzir fotoquimicamente $\mathrm{Se}(\mathrm{VI})$ para $\mathrm{Se}(\mathrm{IV})$ em meio ácido e na presença de $\mathrm{H}_{2} \mathrm{O}_{2}$, com rendimento de $90 \%$. Os autores evidenciaram a necessidade da presença de espécies de sacrifício atuando como doadores de elétrons que, em amostras naturais, poderiam ser as próprias substâncias húmicas que, inclusive, é preciso eliminar para não haver interferência no detector eletroquímico. Desta maneira, o procedimento cumpre as duas funções: de destruição da matéria orgânica e da conversão do Se para uma forma adequada à determinação voltamétrica. Caso o conteúdo de redutor orgânico seja insuficiente, é possível promover a reação mediante adição de glicose. A quantificação seletiva de Se(IV) e $\mathrm{Se}(\mathrm{VI})$, não testada no trabalho citado, seria possível determinando Se antes e depois do foto-tratamento, desde que o material orgânico não afete na etapa de detecção.

Já Boussemart e van den Berg ${ }^{56}$ aproveitaram a foto-oxidação de $\mathrm{Cr}(\mathrm{III})$ para $\mathrm{Cr}(\mathrm{VI})$, num procedimento que permite quantificar separadamente as duas espécies em água de mar. No método proposto, o $\mathrm{Cr}$ (III) é, antes de tudo, seletivamente adsorvido em partículas de sílica, uma escolha imposta pela necessidade de pré-concentrar esta espécie que se encontra em baixas concentrações nas amostras analisadas, ao passo que $\mathrm{Cr}(\mathrm{VI})$ é determinado na fase líquida por voltametria de "stripping" catódico. Numa segunda etapa, o Cr(III) é liberado da sílica para a fase líquida por foto-oxidação e, subseqüentemente, determinado como $\mathrm{Cr}(\mathrm{VI})$. Como somente o $\mathrm{Cr}$ (III) é seletivamente adsorvido na sílica, o $\mathrm{Cr}(\mathrm{VI})$ é solubilizado tão logo ocorra a oxidação.

\section{CONCLUSÕES}

Não há dúvidas sobre o renovado interesse, demonstrado cada vez mais nos últimos anos, pelo uso de técnicas de foto-decomposição no preparo de amostras. Esta redescoberta deve-se, em parte, à grande expansão das técnicas de análise em fluxo, que privilegiam etapas prévias envolvendo o menor risco de pressurização do sistema e, portanto, facilmente integráveis em linha. Como se viu nas seções anteriores, esta feliz associação entre foto-reatores e sistemas em fluxo impulsionou o acoplamento de etapas fotoquímicas com técnicas cromatográficas. Nestas e noutras aplicações, p. ex. métodos eletroquímicos para discriminar entre complexos lábeis e inertes de metais pesados em amostras de água, a irradiação por UV confirmou-se uma importante parceira em procedimentos de especiação química, uma outra área em forte crescimento.

Pode ser observado que vários laboratórios, ao considerarem com maior atenção processos de foto-digestão, apresentaram novas propostas para aumentar a eficiência da técnica, ou para utilizá-la em combinação com outros métodos de abertura de amostras e estender sua gama de aplicabilidade.

Já no caso das etapas de foto-derivatização, é bastante surpreendente constatar como ainda há um número bastante limitado de métodos analíticos, especialmente em fluxo, baseados em reações que, de fato, são bem conhecidas e caracterizadas há muito tempo ${ }^{45}$. Assim, acredita-se que este possa representar um campo fecundo de desenvolvimento de novos métodos, lembrando que à vantagem de serem facilmente implementados em sistemas em fluxo, as reações fotoquímicas, muitas vezes, dispensam o uso de reagentes e são via de regra - mais rápidas que as reações térmica convencionais e podem ser tornadas facilmente mais seletivas, com a escolha do comprimento de onda incidente.

\section{AGRADECIMENTOS}

Os autores expressam seus agradecimentos ao CNPq e à FAPESP pela bolsa de doutoramento de A. Cavicchioli no período de 19982002.

\section{REFERÊNCIAS}

1. Anderson R.; Sample Pretreatment and Separation. Analytical Chemistry by Open Learning, Wiley: Chichester, 1991.

2. Arruda M. A. Z.; Santelli R. E.; Quim. Nova 1997, 20, 638.

3. Bock, R.; A Handbook of Decomposition Methods in Analytical Chemistry, $1^{\text {st }}$ ed., John Wiley and Sons: New York, 1979.

4. Krug, J. F.; Pré-tratamento de amostras, Cena: Piracicaba, 1996.

5. Hanway, R.; Cavicchioli, A.; Kaur, B.; Parsons, J.; Lamb, J. H.; Buckberry, L. D.; Farmer, P. B.; Biomarkers 2000, 5, 252.

6. Batley G. E. Em Trace Element Speciation: Analytical Methods and Problems; Bartey, G. E., ed.; CRC Press: Boca Raton, 1989, p. 43.

7. Pérez-Ruiz, T.; Martínez-Lozano, C.; Tomás, V.; Sanz, A.; Analyst 1996, $121,477$.

8. Golimowski, J.; Golimowska, K.; Anal. Chim. Acta 1996, 325, 111.

9. Wayne, R. P.; Principles and Applications of Photochemistry, Oxford University Press: Oxford, 1988.

10. Phillips, R.; Sources and Applications of Ultraviolet Radiation, Academic Press Inc.: London, 1983.

11. Braun, A. M.; Maurette, M. T.; Oliveros, E.; Photochemical Technology, Wiley: Chichester, 1991.

12. Aguiar, M. A. S; Márquez, K. S. G.; Gutz, I. G. R.; Electroanalysis 2000, 12,742 .

13. Cavicchioli, A.; Gutz, I. G. R.; Anal. Chim. Acta 2001, 445, 127.

14. Campos, M. L. A. M.; Mello, L. C.; Zanette, R.; Sierra, M. M. S.; Bendo, A.; Quim. Nova 2001, 24, 257.

15. Ciccioli, P.; Tappa, R.; Guiducci, A.; Anal. Chem. 1981, 53, 1311.

16. Scholten, A. H. M. T.; Welling, P. L. M.; Brinkman, U. A. Th.; Frei, R. W.; J. Chromatogr. 1980, 199, 239.

17. Achterberg, E. P.; van den Berg, C. M. G.; Anal. Chim. Acta 1994, 291, 213.

18. Barni, B.; Cavicchioli, A.; Riva, E.; Zanoni, L.; Bignoli, F.; Bellobono, I. R.; Gianturco, F.; DeGiorgi, A.; Muntau, H.; Montanarella, L.; Facchetti, S.; Castellano, L.; Chemosphere 1995, 30, 1847.

19. Begerow, J.; Dunemann, L. Em Sampling and Sample Preparation; Stoeppler M., ed.; Springer Verlag: Berlim, 1997, p. 155.

20. Burguera, M.; Burguera, J. L.; Quim. Anal. 1996, 15, 112.

21. Oliveira, A. F; Fatibello-Filho, O.; Talanta 1999, 50, 899.

22. Chmilenko, F. A.; Baklanov, A. N.; Sidorova, L. P.; Piskun, Y. M.; J. Anal. Chem. 1994, 49, 550.

23. Gouveia, S. T.; Fatibello-Filho, O.; Nóbrega, J. A.; J. Braz. Chem. Soc. 2000, 11, 261.

24. Rajeshwar, K.; Ibañez, J. G.; Environmental Electrochemistry: Fundamental and Applications in Pollution Abatement, Academic Press: San Diego, 1997.

25. Brainina, K. Z.; Anal. Chim. Acta 1995, 305, 146.

26. Armstrong, F. A. J.; Williams, P. M.; Strickland, D. H.; Nature 1966, 211, 481.

27. Sipos, L.; Golimowski, J.; Valenta, P.; Nürnberg, P.; Fresenius J. Anal. Chem. 1979, 298, 1.

28. Dorten, W.; Valenta, P.; Nurnberg, H. W.; Fresenius J. Anal. Chem. 1984, 317, 264.

29. Saur, D. Em Sample Preparation Techniques in Voltammetric Analysis; Bruttel, P. A.; Schäfer, J, eds.; Metrhom: Herisau, 1992, p. 5.

30. Labuda, J.; Saur, D.; Neeb, R.; Fresenius J. Anal. Chem. 1994, 348, 312.

31. Kolb, M.; Rach, P.; Schäfer, J.; Wild, A.; Fresenius J. Anal. Chem. 1992, 342,341 .

32. Yokoi, K.; Yakushiji, M.; Hatanaka, M.; Kubono, K.; Koide, T.; Fresenius J. Anal. Chem. 1999, 365, 364.

33. Vega, M.; Pardo, R.; Barrado, E.; de la Fuente, M. A.; del Valle, J. L.; Fresenius J. Anal. Chem. 1994, 350, 139.

34. Benson, R. L.; McKelvie, I. D.; Hart, B. T.; Truong, Y. B.; Hamilton, I. C.; Anal. Chim. Acta 1996, 326, 29.

35. Achterberg, E. P.; van den Berg, C. M. G.; Anal. Chim. Acta 1994, 284 , 463

36. Buldini, P. L.; Cavalli, S.; Mevoli, A.; J. Chromatogr. 1996, 739, 167.

37. Tsalev, D. L.; Sperling, M.; Welz, B.; Analyst 1998, 123, 1703.

38. Tsalev, D. L.; Sperling, M.; Welz, B.; Spectrochim. Acta 2000, 55, 339

39. Ebdon, L.; Hill, S. J.; Jones, P.; Talanta 1991, 38, 607. 
40. Falter, R.; Schöler, H. F.; J. Chromatogr., A 1994, 675, 253.

41. Zhang, X.; Cornelis, R.; De Kimple, J.; Mees, L.; Anal. Chim. Acta 1996, $319,177$.

42. Florian, D.; Knapp, G.; Anal. Chem. 2001, 73, 1515.

43. Ollis, D. F.; Pelizzetti, E.; Serpone, N. Em Photocatalysis: Fundamentals and Applications; Serpone, N.; Pelizzetti, E., eds.; J. Wiley \& Sons: New York, p. 603.

44. Escuriola, M. J.; Morales-Rubio, A.; de la Guardia, M.; Anal. Chim. Acta 1999, 390, 550 .

45. Fitzgerald, J. M.; Analytical Photochemistry and Photochemical Analysis, Marcel Dekker: New York, 1971.

46. Liu, R. M.; Liu, D. J.; Sun, A. L.; Analyst 1992, 117, 1767.

47. Luque-Pérez, E.; Ríos, A.; Valcárcel, M.; Anal. Chim. Acta 1998, 366, 231.
48. Leon, L. E.; Ríos, A.; Luque De Castro, M. D.; Valcárcel, M.; Anal. Chim. Acta 1990, 234, 227.

49. Sánchez-Pedreño, C.; Sierra, M. T.; Sierra, M. I.; Sanz, A.; Analyst 1988, $113,145$.

50. Liu, R. M.; Liu, D. J.; Analyst 1991, 116, 497.

51. Cultrera, R.; Ferrari, G.; Ann. Chim. 1957, 47, 1321.

52. Gil-Torró, I.; García-Mateo, J. V.; Martínez-Calatayud, J.; Anal. Chim. Acta 1998, 366, 241.

53. Liu, R. M.; Liu, D. J.; Sun, A.; Liu, G.; Talanta 1995, 42, 437.

54. Cerdà, A.; Oms, M. T.; Forteza, R.; Cerdà, V.; Analyst 1996, 121, 13.

55. Mattsson, G.; Nyholm, L.; Olin, A.; Örnemark, U.; Talanta 1995, $42,817$.

56. Boussemart, M.; van den Berg, C. M. G.; Analyst 1994, 119, 1349. 